The Nepali Math. Sc. Report,Vol. 38, No.2, 2021:1-12

DOI:10.3126/nmsr.v38i2.42700

\title{
DYNAMIC CONTRAFLOW WITH ORIENTATION DEPENDENT TRANSIT TIMES ALLOWING INTERMEDIATE STORAGE
}

\section{DURGA PRASAD KHANAL ${ }^{1}$, URMILA PYAKUREL ${ }^{2}$, STEPHAN DEMPE $^{3}$}

\author{
${ }^{1}$ Saraswati Multiple Campus, Tribhuvan University, Kathmandu, Nepal \\ ${ }^{2}$ Central Department of Mathematics, Tribhuvan University, Kathmandu, Nepal \\ ${ }^{3}$ Faculty of Mathematics and Computer Science, TU Bergakademie Freiberg, Germany \\ Emails: ${ }^{1}$ durgapsdkhanal@gmail.com, ${ }^{2}$ urmilapyakurel@gmail.com, \\ 3dempe@math.tu-freiberg.de \\ 2 Corresponding author: urmilapyakurel@gmail.com
}

\begin{abstract}
Contraflow is one of the best and widely accepted techniques in evacuation planning problems, where the reversal of arcs is made to increase the amount and decrease the time of flow transmission. At the time of evacuation, if the flow value leaving the source node exceeds the bottleneck capacity of the network, then the storage of excess flow at comparatively safer intermediate nodes can be a milestone to save the life of evacuees. In this paper, we introduce the maximum dynamic contraflow problem in a general network and earliest arrival contraflow problem in two-terminal series-parallel network with asymmetric transit times on anti-parallel arcs. We present polynomial time solution strategie s with orientation dependent transit times by allowing the storage of flow at intermediate nodes.
\end{abstract}

Key Words: Maximum flow, earliest arrival flow, intermediate storage, contraflow, symmetric and asymmetric transit times.

2010 Mathematics Subject Classification. Primary: 90B10, 90C27, 68Q25; Secondary: 90B06, 90B20.

\section{INTRODUCTION}

Network flow over time problems, also known as dynamic flow problems, deal with the transshipment of flow from one point to other using running time i.e., traversal time. Ford and Fulkerson are the pioneers of this problem which has been introduced more than sixty years ago, 9. Maximum dynamic flow problem concerns the shifting of maximum amount of flow from the origin node (source) to the destination node (sink) in the given time horizon. Evacuation planning problems can be modeled as the maximum dynamic flow problem, which is also applicable in transportation management, communication system and production planning etc. Similarly, earliest arrival flow problem treats with the maximization of flow at each time step, [1]. For the EAF problem, Minieka [21] and Wilkinson 29] provided the algorithms by adopting the successive shortest path augmenting algorithm which needed exponential time. In a two-terminal general network, Pyakurel and Dhamala [25] presented optimal solution to the earliest arrival flow problem for discrete and continuous time settings. The detailed illustrations of maximum flow can be found in the books 11, 10, book series of [20, survey papers [2, 19, 7, 14, and the references therein.

Existance of the excess flow occures if the amount of flow leaving the source node is greater than the minimum cut capacity. In this case, the excess flow cannot reach the destination node and the storage of such flow at appropriate intermediate nodes is an important issue. The maximum flow problem with intermediate storage is a very relevant task in large scale disaster management problems. By introducing

Accepted/Published Online December, 2021 
the concept of intermediate storage, Pyakurel and Dempe 23] provided the maximum static and maximum dynamic flow problems and presented the polynomial time solution strategies to solve the problems. Not only in disaster management and evacuation planning, transshipment of flow with intermediate storage is highly applicable for different demand-supply chains like commodity supply, electricity distribution, water supply and so on.

In a two-way network topology, contraflow (lane reversal) means the reversing of the direction of arcs to increase the amount of flow transmission and reduce the total traversal time. Rebennack et al. [27] presented a polynomial time algorithm to solve the maximum flow problem in two-terminal network by reverting the direction of arcs at time zero and kept fixed afterwards. Various problems with contraflow can be found in [3, 4, 17, 18. The maximum dynamic contraflow (MDCF) problem with intermediate storage and its polynomial solution can be found in Pyakurel and Dempe 23. By reverting only necessary arcs, Dhamala et al. 5] presented algorithms to solve the quickest multi-commodity contraflow problem, where given amount of flow is to be transshipped in minimum possible time. The maximum multi-commodity flow over time problem with partial contraflow is presented by Pyakurel et al. [26]. Similarly, in general as well as two-terminal series-parallel networks, efficient algorithms for the universal maximum flow and contraflow problems with intermediate storage can be found in Pyakurel and Dempe 24]. For these contraflow problems, networks are considered with symmetric transit times in anti-parallel arcs.

For contraflow network with asymmetric transit times, if the transit time of reversal arc depends on the orientation of arc, then it is known as orientation dependent transit times (ODTT). Different transit times on anti-parallel arcs exist in the real network scenario of private as well as public transportation due to the speed limits, time card system, condition of roads and government policies. To illustrate ODTT with an example, consider a two-way dynamic network with asymmetric transit times on arcs as presented in Figure 1(i). The numbers on each arc represent the capacity $u$ and transit time $\tau$, whereas numbers on the nodes are storage capacities $b$. The other four figures are the possible auxiliary networks with ODTT. Figure 1(ii) is formed by taking directions along $(x, y)$ and $(x, t)$, whereas Figure 1(iii) with directions along $(y, x)$ and $(x, t)$. Similarly, Figure 1(iv) is formed by taking directions along $(x, y)$ and $(t, x)$, whereas Figure 1 ( v) is with directions along $(y, x)$ and $(t, x)$. Nath et al. 22. presented the concept of contraflow with orientation dependent transit times, where asymmetric transit times of reversed lanes in general form is considered. They have presented strongly polynomial time algorithms to solve the maximum dynamic and quickest contraflow problems in single source single sink network. Gupta et al. [12] extended the analytical solution of generalized dynamic contraflow problem by considering the asymmetric transit times on arcs and presented an efficient algorithm to solve the problem. The earliest arrival transshipment contraflow problem in multi-source single sink network and the prioritized maximum dynamic partial contraflow problem in single source multi-sink network by considering the non-symmetric transit times on the arcs can be found in 13. Similarly, for maximum multi-commodity contraflow problems with symmetric and asymmetric transit times, Khanal et al. [16] presented the pseudo polynomial time algorithms to solve the problems.

In this paper, we present the models for static as well as dynamic flow by incorporating the intermediate storage of the flow. We introduce the MDCF problem with ODTT by allowing the storage of excess flow at intermediate nodes and present a polynomial time algorithm to solve it. We also introduce an earliest arrival contraflow $(\mathrm{EACF})$ problem with ODTT and propose a solution in polynomial time complexity by allowing the intermediate storage.

The paper is organized as follows. In Section 2, we present a contraflow network and its auxiliary network with symmetric as well as asymmetric transit times. We also formulate static and dynamic flow models with intermediate storage. The MDCF with ODTT and EACF with ODTT are presented in Section 3 and Section 4, respectively, which permit the storage of flow at intermediate nodes. We conclude the paper in Section 5. 


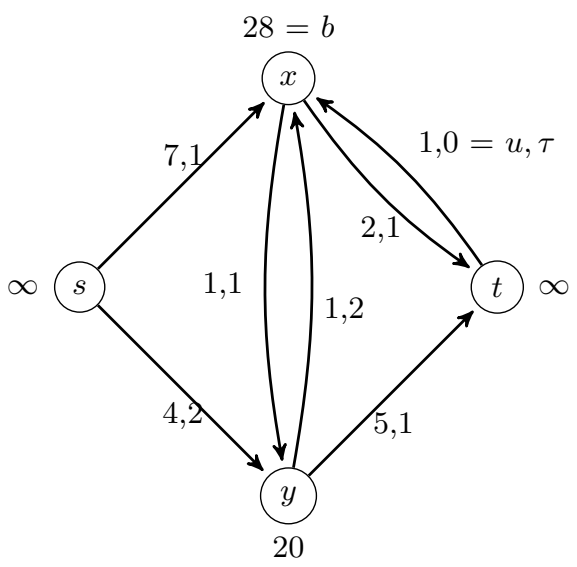

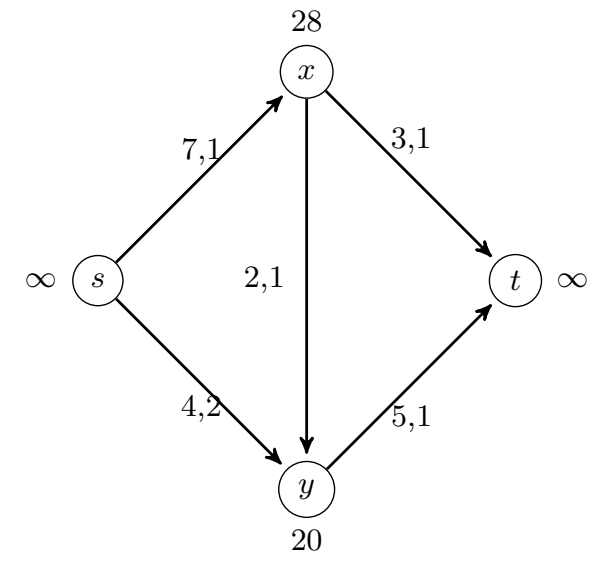

(ii)

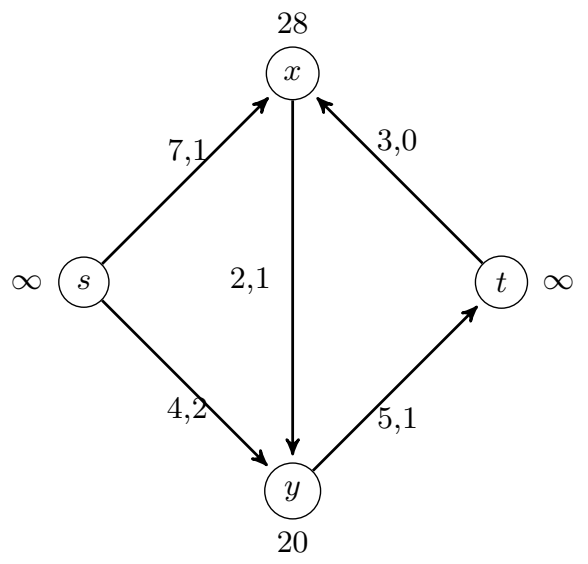

(iv)

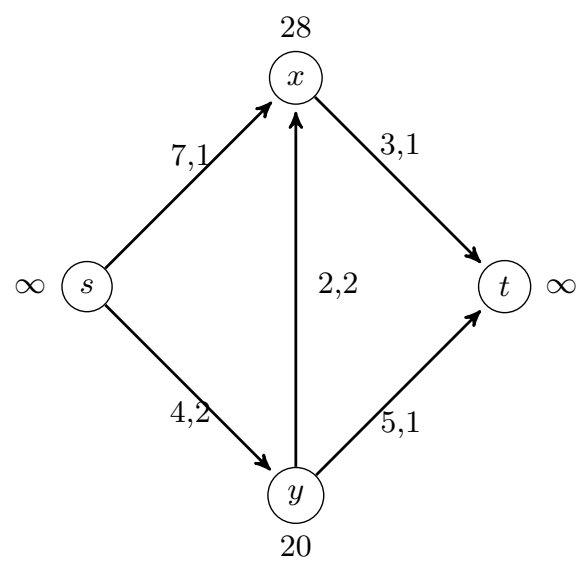

(iii)

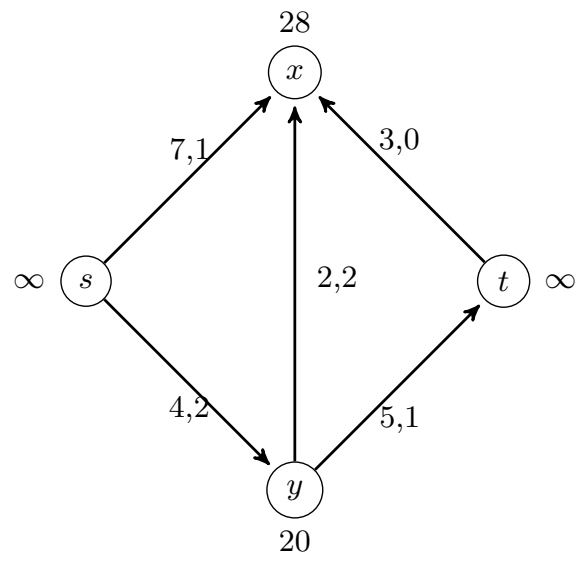

(v)

FIGURE 1. (i) represents a contraflow network with asymmetric transit times and (ii)-(v) represent possible orientation dependent auxiliary networks of (i). Numbers aside the nodes are the storage capacities.

\section{Basic Terminologies And Flow Models}

2.1. Contraflow Network. Consider a two-way network topology $\mathcal{N}=(V, A, u, b, \tau, s, t, T)$, where $V$ represents the set of nodes and $A \subseteq V \times V$ represents the set of arcs. Let $|V|=n$ and $|A|=m$. Here, $s \in V$ and $t \in V$ are the source (origin) and sink (destination) nodes and $I=V \backslash\{s, t\}$ represents the set of intermediate nodes. Each arc $a=(v, w) \in A$ with head $(a)=w$ and $\operatorname{tail}(a)=v$ has a capacity function 
$u: A \rightarrow \mathcal{R}^{+}$that limits the flow on arc and a non-negative transit time function $\tau: A \rightarrow \mathcal{R}^{+}$that measures the transmission time from $v$ to $w$. Similarly, $\overleftarrow{a}=(w, v)$ is an oppositely directed arc of $a=(v, w)$ with capacity $u_{\overleftarrow{a}}$ and transit time $\tau_{\overleftarrow{a}}$. Let $b: V \rightarrow \mathcal{R}^{+}$represents the storage capacity function of the nodes. We denote the set of outgoing $\operatorname{arcs}$ from node $v$ and incoming $\operatorname{arcs}$ to node $v$ by $\delta^{\text {out }}(v)$ and $\delta^{\text {in }}(v)$, respectively. The time period $T$ given in advanced is denoted by $\mathcal{T}=\{0,1, \ldots, T\}$ in discrete time settings. In static flow, the time parameters are absent.

Auxiliary Network with Symmetric Transit Times. Consider a dynamic network $\mathcal{N}$ having antiparallel arcs of symmetric transit times i.e., $\tau_{a}=\tau_{\overleftarrow{a}}$. The auxiliary network of given network $\mathcal{N}$ is denoted by $\overline{\mathcal{N}}=(V, \bar{A}, \bar{u}, b, \bar{\tau}, s, t, T)$, containing undirected edges $\bar{A}=\{(v, w):(v, w)$ or $(w, v) \in A\}$. The capacity $\bar{u}_{a}$ of an arc is the sum of capacities of anti-paralled $\operatorname{arcs} a$ and $\overleftarrow{a}$, i.e., $\bar{u}_{a}=u_{a}+u_{\overleftarrow{a}}$, where $u_{a}=0$ if $a \notin A$. Similarly, the transit time of an arc in this network is obtained as

$$
\bar{\tau}_{a}= \begin{cases}\tau_{a} & \text { if } a \in A \\ \tau_{\overleftarrow{a}} & \text { otherwise }\end{cases}
$$

All others parameters are as same in $\mathcal{N}$.

Auxiliary Network with ODTT. In a two-way dynamic network, if the transit times on anti-parallel arcs are not identical, then it is known as the network with asymmetric transit times. In contraflow configuration, the flow on anti-parallel arcs traverses in one of the directions but not the both directions. In auxiliary network, if we consider the transit time of the arc depending on its orientation, then it is defined as the contraflow with orientation dependent transit times. For a two-way dynamic network $\mathcal{N}$ with asymmetric transit time on arcs, an auxiliary network $\overline{\mathcal{N}}=(V, \bar{A}, \bar{u}, b, \bar{\tau}, s, t, T)$ is constructed in such a way that $\bar{A}$ contains the undirected arcs obtained by reverting the direction of $\operatorname{arcs} \overleftarrow{a}$ at time zero. The arc capacity $\bar{u}_{a}$ and transit time $\bar{\tau}_{a}$ are obtained by $\bar{u}_{a}=u_{a}+u_{\overleftarrow{a}}$ where $u_{a}=0$ if $a \notin A$ and

$$
\bar{\tau}_{a}= \begin{cases}\tau_{a} & \text { if the orientation of arc is along } a \\ \tau_{\overleftarrow{a}} & \text { if the orientation of arc is along } \overleftarrow{a} \text { or } a \notin A\end{cases}
$$

It is to be noted that if there is no arc along the orientation of auxiliary network, then transit time of auxiliary arc is taken as the symmetric reversal of the opposite arc. Throughout the paper, our assumption is that the storage capacity of terminals are significant enough, i.e., $b_{s}=b_{t} \leq \infty$ and the intermediate nodes have finite storage capacity. The excess flow at intermediate nodes exists if and only if the sum of outgoing arc capacities of an intermediate node $v \in I$ is less than the sum of incoming arc capacities. Moreover, the storage capacity $b_{v}$ of intermediate node $v \in I$ should be at least the sum of incoming arc capacities of $v$ for unique solution. Otherwise, it provides an alternate optimal solution (see explanation of Figure 2 .

2.2. Static Flow Model with Intermediate Storage. The static flow $\psi$ with intermediate storage on a given two-way network $\mathcal{N}=(V, A, u, b, c, s, t)$ is the collection of non-negative arc flow functions $\psi_{a}: A \rightarrow \mathcal{R}^{+}$ and the excess flow functions $\psi_{v}: I \rightarrow \mathcal{R}^{+}$. The static flow model with intermediate storage is the network flow satisfying the conditions 2.1-2.4. As in [23, the mathematical formulation of the maximum static flow with intermediate storage, as a linear programming problem, is as follows.

$$
\max |\psi|=\sum_{a \in \delta^{\text {out }}(s)} \psi_{a}=\sum_{a \in \delta^{\text {in }}(t)} \psi_{a}+\sum_{v \in I: b_{v} \geq 0} \psi_{v}
$$

such that,

$$
\begin{aligned}
\sum_{a \in \delta^{i n}(v)} \psi_{a} & -\sum_{a \in \delta^{\text {out }}(v)} \psi_{a}=\psi_{v} \forall v \in I \\
0 \leq \psi_{a} & \leq u_{a} \quad \forall a \in A \\
0 \leq \psi_{v} & \leq b_{v} \quad \forall v \in I
\end{aligned}
$$

Equation 2.1] is an objective function that is to maximize the total outflow from the source which must be equal to the sum of inflow at sink and the total excess flow at intermediate nodes. Here, $|\psi|$ denotes the total value of the flow. The excess flow at intermediate nodes is represented by Equation 2.2 and 
Equation 2.3 represents the boundedness of the flow on each arc. The left inequality in constraint 2.4 represents the non-conservation of flow where the right inequality shows the boundedness of the excess flow by the storage capacity of the node. For existence of unique solution, the storage capacity of intermediate node $v \in I$ must be at least the sum of incoming arc capacities to $v$. Mathematically, it is represented as $\sum_{a \in \delta^{i n}(v)} u_{a} \leq b_{v} \quad \forall v \in I$. We define the cost $c$ of static flow $\psi$ associated with arc $a$ as

$$
c(\psi)=\sum_{a \in A} c_{a} \psi_{a} .
$$

At the time of an emergency, the general assumption is that the flow towards the danger zone is almost empty and so we reverse the direction of arcs towards the safe zone (destination). After reversing the direction of arcs using orientation dependent cost, the contraflow model can be obtained replacing $a \in A$ by $a \in \bar{A}$ and $u_{a}$ by $\bar{u}_{a}$. At last, the flow obtained in the auxiliary network is transformed to the original network.

2.3. Dynamic Flow Model with Intermediate Storage. The flow over time function $\Phi$ defined on a dynamic network $\mathcal{N}$ with arc traversal time $\tau$ is the collection of non-negative arc flow function $\Phi_{a}$ : $A \times \mathcal{T} \rightarrow \mathcal{R}^{+}$and the excess flow function $\Phi_{v}: I \times \mathcal{T} \rightarrow \mathcal{R}^{+}$. The dynamic contraflow model with ODTT $\tau$ in $\mathcal{N}$ allowing intermediate storage is a network flow satisfying the conditions 2.5-2.8. As in 23], the mathematical model for the maximum dynamic flow allowing the intermediate storage, with linear programming formulation, is as follows.

$$
\max |\Phi|=\sum_{a \in \delta^{\text {out }}(s)} \sum_{\theta=0}^{T} \Phi_{a}(\theta)=\sum_{a \in \delta^{\text {in }}(t)} \sum_{\theta=\tau_{a}}^{T} \Phi_{a}\left(\theta-\tau_{a}\right)+\sum_{v \in I: b_{v} \geq 0} \Phi_{v}(T)
$$

such that,

$$
\begin{aligned}
\sum_{a \in \delta^{\text {in }}(v)} \sum_{\beta=\tau_{a}}^{\theta} \Phi_{a}\left(\beta-\tau_{a}\right) & -\sum_{a \in \delta^{\text {out }}(v)} \sum_{\beta=0}^{\theta} \Phi_{a}(\beta)=\Phi_{v}(\theta) \quad v \in I, \theta \in \mathcal{T} \\
0 \leq \Phi_{a}(\theta) & \leq u_{a} \quad \forall a \in A, \theta \in \mathcal{T} \\
0 \leq \Phi_{v}(\theta) & \leq b_{v} \quad \forall v \in I, \theta \in \mathcal{T}
\end{aligned}
$$

In given time horizon $T$, the objective function in Equation 2.5 refers to the maximization of the total flow out from the source which must be equal to the sum of inflow at sink and the total excess flow at intermediate nodes. The excess flow stored at intermediate nodes is represented by Equation 2.6. Similarly, the constraint in (2.7) represents the boundedness of the arc flow by its capacity at each time step $\theta$. The left inequality of the constraint in 2.8) represents the non-conservation of flow and its right inequality represents the boundedness of the excess flow at the intermediate node by its storage capacity. The necessary (lower bound) and sufficient (upper bound) storage capacity of each intermediate node $v \in I$ is $\sum_{a \in \delta^{i n}(v)} u_{a} \leq b_{v} \leq T \sum_{a \in \delta^{i n}(v)} u_{a} \quad \forall v \in I$. The lower bound is essential for the existence of unique solution. The cost $c$ of discrete dynamic flow $\Phi$ is defined as

$$
c(\Phi)=\sum_{a \in A} c_{a} \sum_{\theta=0}^{T} \Phi_{a}(\theta) .
$$

For EACF problem with ODTT by allowing intermediate storage, the linear programming formulation is as follows.

$$
\max |\Phi(\theta)|=\sum_{a \in \delta^{\text {out }}(s)} \sum_{\beta=0}^{\theta} \Phi_{a}(\beta)=\sum_{a \in \delta^{\text {in }}(t)} \sum_{\beta=\tau_{a}}^{\theta} \Phi_{a}\left(\beta-\tau_{a}\right)+\sum_{v \in I: b_{v} \geq 0} \Phi_{v}(\theta)
$$

subject to the constraints $2.6-2.8$.

As in static case, if we replace $a \in A, \tau_{a}$ and $u_{a}$ in above model by $a \in \bar{A}, \bar{\tau}_{a}$ and $\bar{u}_{a}$, respectively, then the dynamic contraflow model can be obtained with orientation dependent transit times, where the traversal time of the arcs in auxiliary network is depending on its orientation. 
While shifting the flow from the source, first priority is given to the sink because the assumption is that the sink is most appropriate place with respect to location and infrastructure. The priority of intermediate nodes is set with farther-in-distance-higher-in-priority. We fix the priority of nodes before the contraflow configuration because the alternative lane reversals in the network grows exponentially with the number of arc on the network which increases its computational complexity.

For the uniqueness of the solution, the minimum storage capacity of intermediate nodes should be at least the sum of incoming arc capacities i.e., $b_{v} \geq \sum_{a \in \delta^{i n}(v)} u_{a}$. We now verify it by the following example.

Example 1. Consider the network presented in Figure 2 with unit cost/transit time on each arc except $(s, w)$. Let the cost of arc $(s, w)$ be zero. The first priority is given to the sink node. Due to equal cost from the source, second priority is given to $v$ or $y$, the third priority is for $u$ or $x$ and fourth priority is given to $w$. Firstly, we consider 2 units of storage capacity in each intermediate node. If the flow is sent to the sink through paths $s-u-v-w-t$ and $s-w-t$, then $7,2,2,2,0$ and 1 units of flow can be stored at prioritized nodes $t, v, y, u, x$ and $w$, respectively. Again, by sending the flow to the sink through paths $s-x-y-w-t$ and $s-w-t$, the amount of flow stored at prioritized nodes $t, v, y, u, x$ and $w$ are $7,2,0,2,0$ and 2 units, respectively. So, in previous pattern of flow, 7, 4, 2, and 1 units are stored at first, second third and fourth prioritized nodes whereas 7, 2, 2, and 2 units are stored at respective prioritized nodes in case of later pattern, respectively. Thus, instead of a unique optimal solution it provides an alternate optimal solution. Secondly, if we consider the storage capacity of 10 units in each intermediate node, then 7, 5, 2 and 0 units of flow can be stored at first, second, third and fourth prioritized nodes, respectively, in either of the flow patterns.

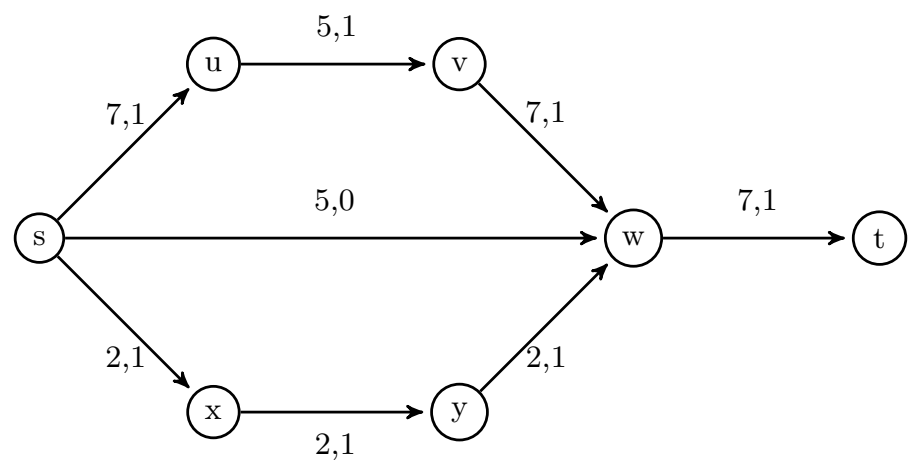

FIgURE 2. Network with capacity and unit cost/transit time on each arc.

\section{MDCF with ODTT Allowing Intermediate Storage}

In this section, we introduce the MDCF problem in which time to traverse through the arcs in auxiliary network are orientation dependent and the storage of flow at intermediate nodes is allowed. We present a polynomial time solution strategy to solve the problem.

Problem 1. For a given two-way dynamic network $\mathcal{N}=(V, A, u, b, \tau, s, t, T)$ with auxiliary network $\overline{\mathcal{N}}$, the $M D C F$ problem with ODTT by allowing the storage of flow at intermediate nodes is to maximize the flow out from the source $s$ which is to be transshipped to the sink $t$ via $s$-t paths together with the storage of maximum excess flow at the intermediate nodes with storage capacity $\sum_{a \in \delta^{i n}(v)} \bar{u}_{a} \leq b_{v} \leq T \sum_{a \in \delta^{i n}(v)} \bar{u}_{a}$ within the time horizon $T$, where the reversal of the arcs are made at time zero and transit times on auxiliary network are orientation dependent.

To solve the problem, the priority order of the nodes is fixed on the basis of farther-in-distance-higherin-priority order as described in Pyakurel and Dempe [23], where distance of each intermediate node from the source is obtained by using Dijkstra's algorithm ([8]) by considering transit time as cost. That is, $\forall v_{1}, v_{2} \in I$ 
if $d_{\left(s, v_{1}\right)}>d_{\left(s, v_{2}\right)}$, then $v_{1}$ is more relevant and highly prioritized than $v_{2}$ and is denoted by $v_{1} \succ v_{2}$. We then transform the given network into an auxiliary network $\overline{\mathcal{N}}$ with orientation dependent transit times. The flow is send from the source with first priority to the sink and then successive order of prioritized intermediate nodes.

For each prioritized node $v \in I$, we create the dummy port $v^{\prime}$ with capacity $u_{\left(v, v^{\prime}\right)}=b_{v}=b_{v^{\prime}}$, where $u_{\left(v, v^{\prime}\right)}$ is the arc capacity of dummy arc $\left(v, v^{\prime}\right)$. The transit time of dummy arc is $\tau_{\left(v, v^{\prime}\right)}=0$. Every dummy port $v^{\prime}$ has the same priority order as $v$ has. The modified auxiliary network $\overline{\mathcal{N}}^{\prime}=\left(V^{\prime}, \bar{A}^{\prime}, \bar{\tau}, \bar{u}, b, s, D\right)$ is constructed having single source $s$ and multiple sink $D=\left\{t \cup\left\{v^{\prime}\right\}\right\}$, where $\left\{v^{\prime}\right\}$ is the collection of dummy ports. Here, $V^{\prime}=V \cup\left\{v^{\prime}\right\}$, and $\bar{A}^{\prime}=\bar{A} \cup\left\{\left(v, v^{\prime}\right)\right\}$. For an instance, if $t \succ v_{1} \succ \cdots \succ v_{r}$ be priority order of intermediate nodes and sink, then $D=\left\{t=v_{0}^{\prime}, v_{1}^{\prime}, \ldots, v_{r}^{\prime}\right\}$. We construct a new modified network by introducing a super sink $t^{*}$ having sufficiently large (infinite) storage capacity and join each $v^{\prime} \in D$ by an arc with zero transit time and infinite capacity (See Figure 3). Using lexicographic maximum flow, the maximum dynamic flow with intermediate storage in an auxiliary network is obtained as in 23], where the reversal of arcs are orientation dependent. Finally, flow in the auxiliary network is transformed into the original network.

We present a polynomial time algorithm to solve the problem as follows.

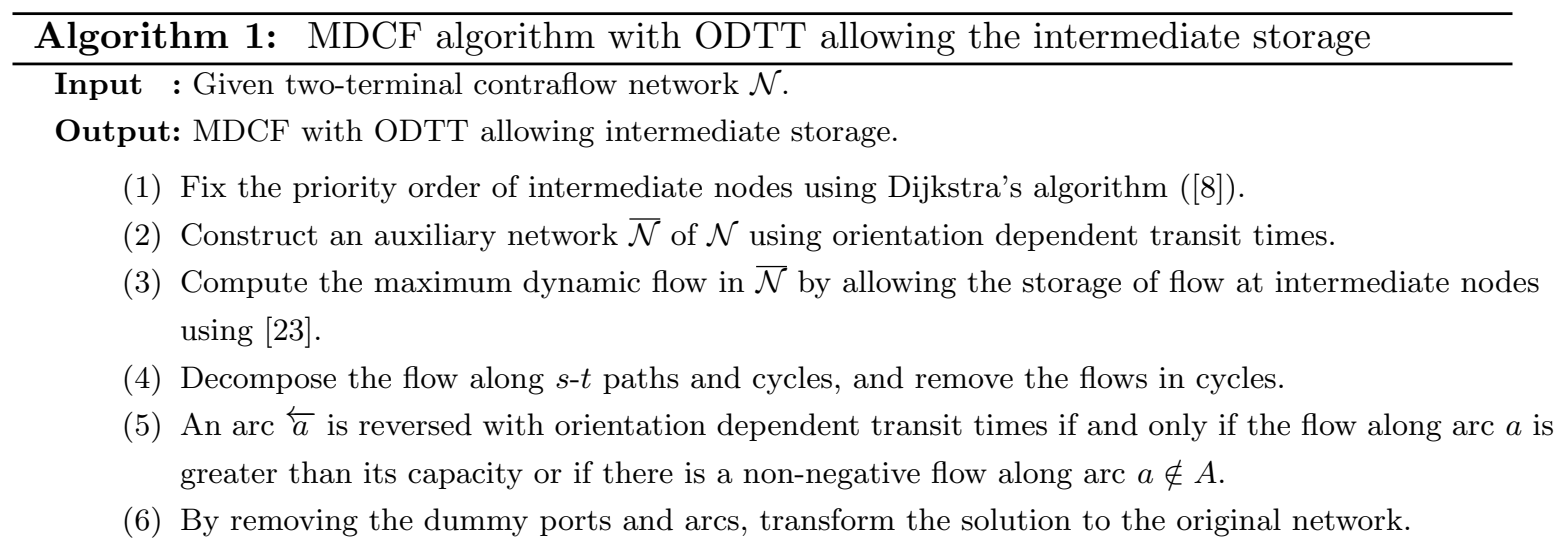

Theorem 3.1. Algorithm 1 provides the feasible solution to MDCF problem with ODTT by allowing the storage of flow at intermediate nodes optimally.

Proof. At first, we show the feasibility and then the optimality of Algorithm 1 Steps (1) and (2) are the use of Dijkstra's algorithm and the construction of an auxiliary network, respectively, which are feasible. In Step 3 finding the maximum dynamic flow with intermediate storage is feasible by [23. As steps (4), (5) and (6) are construction of cycle free paths, condition of arc reversals and transformation of solution, which are feasible. So, Algorithm 1 provides the feasible solution. Next, the optimality of algorithm is dominated by the optimality of Step [3], which is optimal as proven by Pyakurel and Dempe [23. So, Algorithm 1 solves the MDCF with ODTT by allowing the storage of flow at intermediate nodes optimally.

Theorem 3.2. MDCF problem with ODTT can be solved by Algorithm 1 allowing the intermediate storage in polynomial time complexity.

Proof. The time complexity of Dijkstra's algorithm in Step 1 is $O(m \log n)$ and that of shorting algorithm is $O(n \log n)$. Steps (2) and (4) of Algorithm 1 can be obtained in linear time. Step (3) calculates a priority based maximum dynamic flow in $s-D$ network in $O(n \times M C F(n, m))$ time, where $M C F(n, m)$ represents the time complexity of single source single sink minimum cost flow in original network, which can be solved within $O(m \log n(m+n \log n))$ time. So, Algorithm 1 solves the MDCF problem with ODTT by allowing the intermediate storage in polynomial time complexity. 
Example 2. Consider the dynamic contraflow network given in Figure 1(i) with time horizon $T=4$. Using Dijkstra's algorithm [8], the priority ordering is set as $t \succ y \succ x$. Four different auxiliary networks with ODTT are presented in Figure 1.(ii)-(v)]. Figure[1(ii) represents an auxiliary network with orientation along $(x, y)$ and $(x, t)$ which sends 19,8 and 13 units of flow at $t, y$ and $x$ in $T=4$, whereas Figure 1 (iii) is with orientation along $(y, x)$ and $(x, t)$ which sends 17,4 and 19 units of flow at $t, y$ and $x$ in $T=4$, respectively. Similarly, Figure 1 (iv) is obtained by taking orientation in auxiliary network along $(x, y)$ and $(t, x)$ which sends 10,8 and 22 units of flow at $t, y$ and $x$ in $T=4$, whereas Figure 1(v) is with orientation along $(y, x)$ and $(t, x)$ which sends 8,4 and 28 units of flow at $t, y$ and $x$ in $T=4$, respectively. In each orientation, total 40 units of flow is pushed from source in given time horizon but Figure 1 (ii) is only the network which pushes maximum flow at sink and the prioritized farthest intermediate node. Thus, the best solution with ODTT is obtained from Figure 1(ii), whose new modified network with super sink is presented in Figure 3 The detailed illustration of flow in this network is given in Table 1 below.

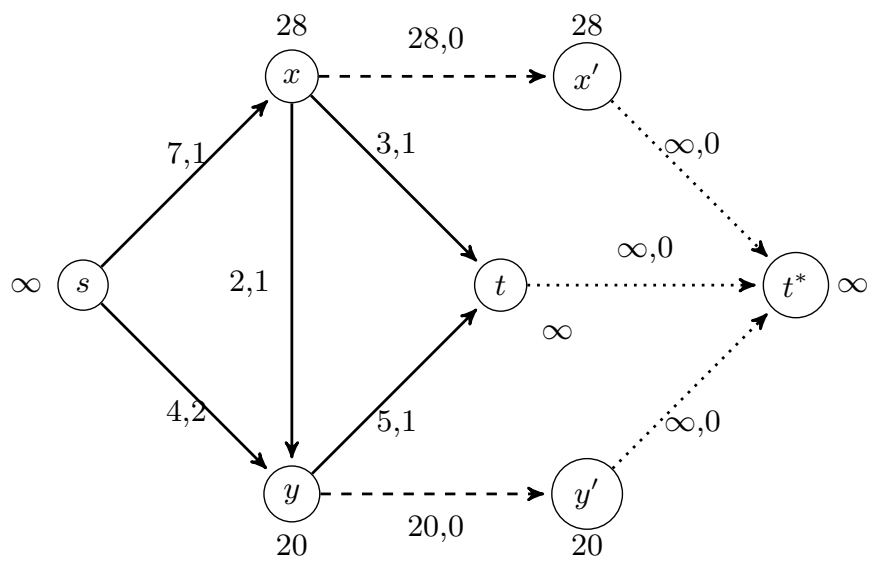

Figure 3. New modified network of Figure 1(ii).

TABLE 1. MDCF with ODTT allowing intermediate storage at time $\theta$.

\begin{tabular}{|c|c|c|c|c|c|}
\hline \hline Path & Start time & \multicolumn{3}{|c|}{ flow at } & Reaching time \\
\cline { 3 - 6 } & at $s$ & $x$ & $y$ & $t$ & at last node \\
\hline$s-x-t$ & $\theta=0$ & 2 & $\times$ & 3 & $\theta=2$ at $t$ \\
\cline { 2 - 6 } & $\theta=1$ & 2 & $\times$ & 3 & $\theta=3$ at $t$ \\
\cline { 2 - 6 } & $\theta=2$ & 2 & $\times$ & 3 & $\theta=4$ at $t$ \\
\cline { 2 - 6 } & $\theta=3$ & 7 & $\times$ & $\times$ & $\theta=4$ at $x$ \\
\hline$s-y-t$ & $\theta=0$ & $\times$ & 0 & 4 & $\theta=3$ at $t$ \\
\cline { 2 - 6 } & $\theta=1$ & $\times$ & 0 & 4 & $\theta=4$ at $t$ \\
\cline { 2 - 6 } & $\theta=2$ & $\times$ & 4 & $\times$ & $\theta=4$ at $y$ \\
\hline$s-x-y-t$ & $\theta=0$ & 0 & 1 & 1 & $\theta=3$ at $t$ \\
\cline { 2 - 5 } & $\theta=1$ & 0 & 1 & 1 & $\theta=4$ at $t$ \\
\cline { 2 - 5 } & $\theta=2$ & 0 & 2 & $\times$ & $\theta=4$ at $y$ \\
\hline & Total & 13 & 8 & 19 & Total=40 \\
\hline
\end{tabular}

\section{EACF with OdTt Allowing Intermediate Storage}

We aim to introduce an EACF problem in a two-terminal series-parallel network by allowing the storage of flow at intermediate nodes and adopting the orientation dependent transit times in auxiliary network. To solve the problem, we present a polynomial time solution strategy. 
Problem 2. For a given two-terminal series-parallel network $\mathcal{N}=(V, A, u, b, \tau, s, t, T)$ with auxiliary network $\overline{\mathcal{N}}$, an EACF problem with ODTT permitting the storage of flow at the intermediate nodes is to maximize the flow leaving the source $s$ at each time step $\theta$, that is to be transshipped to the sink $t$ via s$t$ paths together with the storage of maximum excess flow at the intermediate nodes with storage capacity $\sum_{a \in \delta^{i n}(v)} \bar{u}_{a} \leq b_{v} \leq T \sum_{a \in \delta^{i n}(v)} \bar{u}_{a}$ within time horizon $T$, where the reversal of the arcs are made at time zero and transit times on auxiliary network are orientation dependent.

To solve the problem, we first transform the given $s$ - $t$ network into $s$ - $D$ network by creating dummy ports as described in Section 3 Considering $t^{*}$ as the super sink and joining it to each node in $D$ with infinite capacity and zero transit time, it is reduced to the single source single sink $s-t^{*}$ network. As in Pyakurel and Dempe [24], the minimum cost circulation flow with time bound of Ruzika et al. [28] provides the solution of EACF problem with ODTT by allowing the intermediate storage of the flow in polynomial time complexity, where transit times are orientation dependent. Hereafter, we present an algorithm which provides the solution to an EAF Problem 2 with ODTT by allowing the intermediate storage.

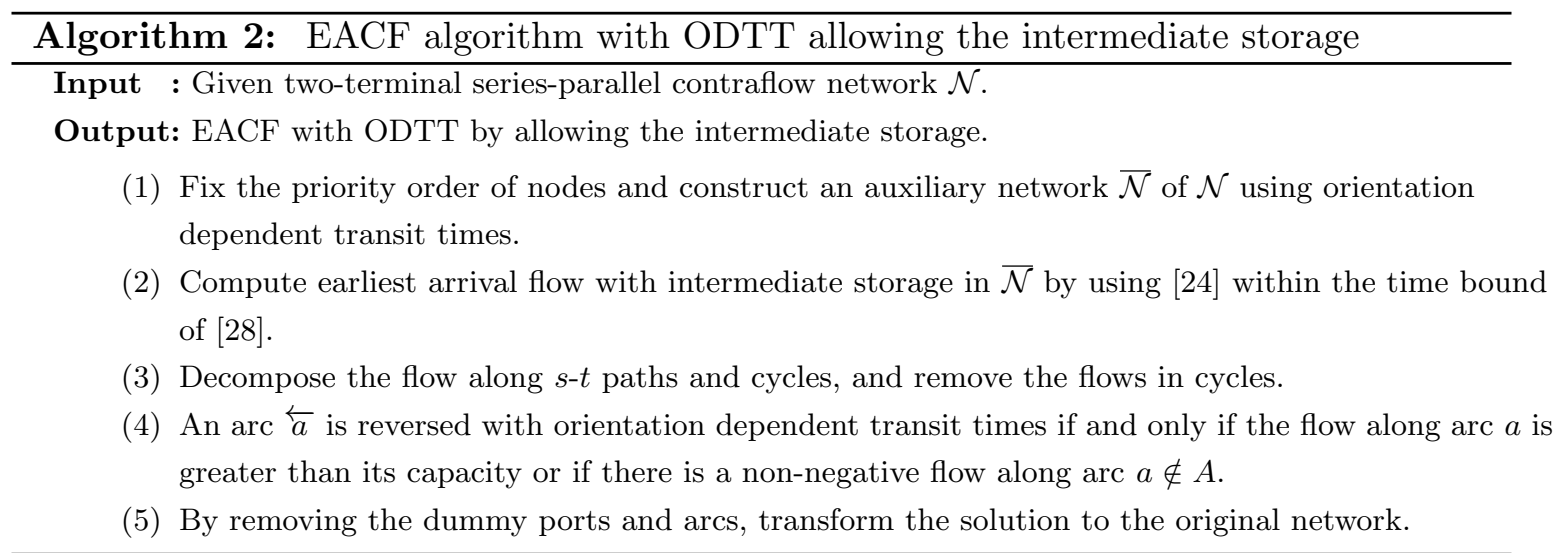

Theorem 4.1. The optimal solution to the EACF problem with ODTT by allowing the intermediate storage can be obtained by using Algorithm 2 .

Proof. The feasibility and optimality of Steps (1), 33, 44 and (5) can be obtained as in Theorem 3.1 In step (2), we compute the earliest arrival flow in auxiliary network with orientation dependent transit times by using algorithm of Pyakurel and Dempe 24] with time bound of Ruzika et al. 28, each providing optimal flow. So Algorithm 2 provides optimal solution to EACF with ODTT by allowing the intermediate storage.

The computational time bound of Step (2) determines the overall time complexity of Algorithm 2 The minimum cost flow problem in series-parallel graph can be solved in $O(n m+m \log m)$ time for single source sink network. So EACF problem with ODTT by allowing intermediate storage can be solved in $O(n \times(n m+m \log m))$ time.

Theorem 4.2. Algorithm 2 solves the EACF problem with ODTT by allowing the intermediate storage in polynomial time.

Example 3. Consider a two-terminal series-parallel contraflow network that is given in Figure 4(i), where numbers aside the nodes represent the storage capacity of nodes. The priority ordering of nodes with respect to the distance is $t \succ z \succ y \succ x \succ w$ (as the shortest distance of $z$ and $y$ from $s$ are same, priority of these two nodes may interchange). The auxiliary network with orientation dependent transit times is constructed in Figure 4 (ii). Let the time horizon be $T=5$. Then, the total amount of earliest arrival flow at $t, z, y, x$ and $w$ in time horizon $T=5$ are $30,9,2,7$ and 16 units, respectively. 


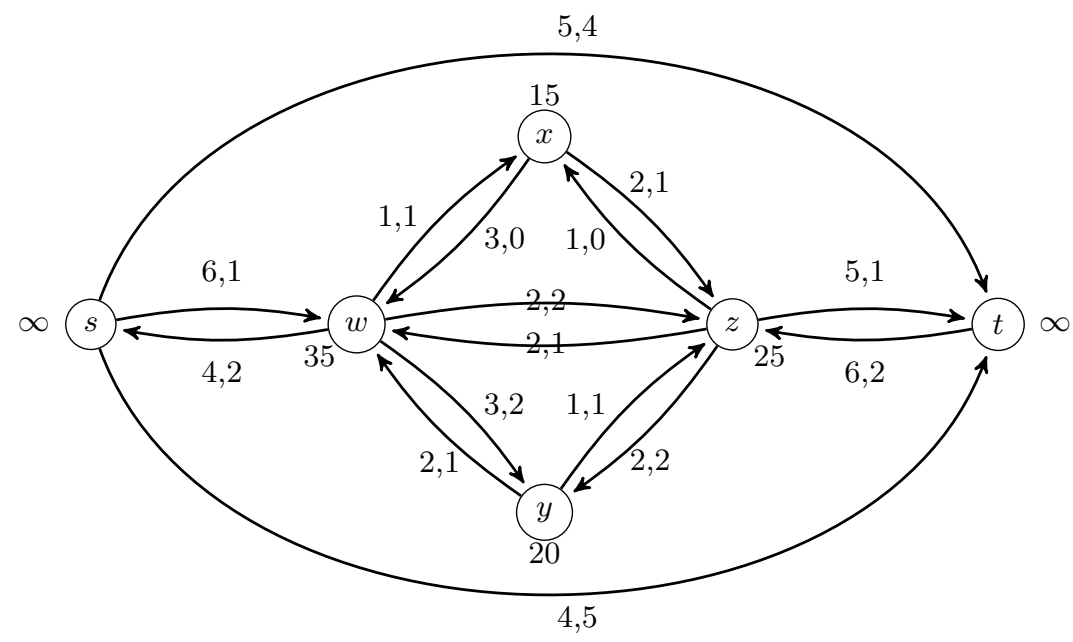

(i) Two-way series-parallel network with capacity, transit time on arc

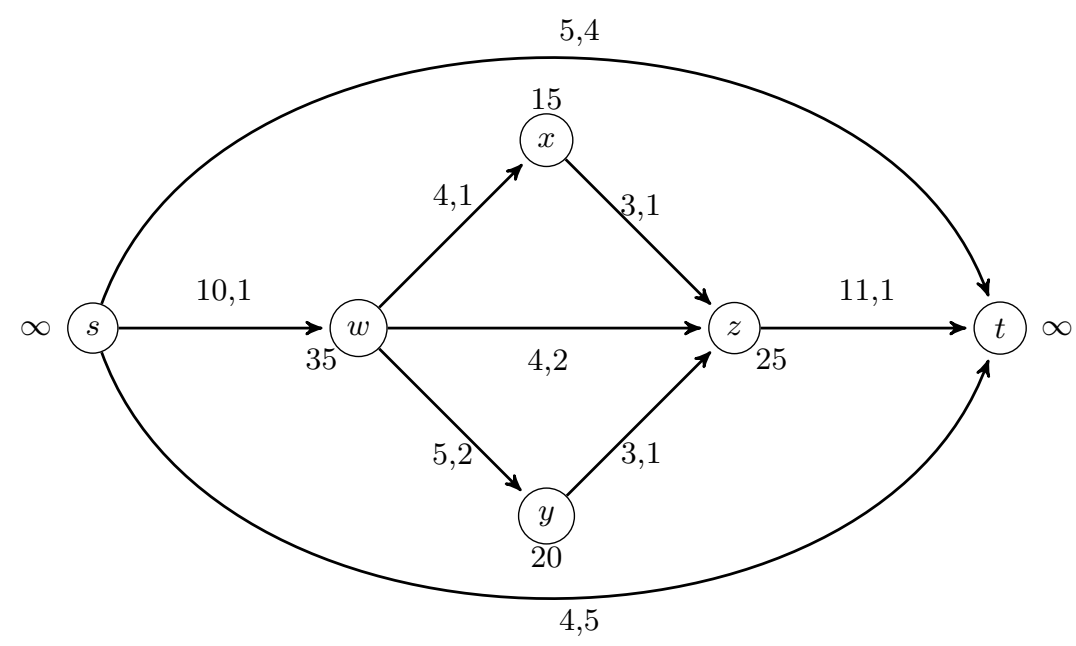

(ii) Auxiliary network with capacity, transit time on arc

FiguRE 4. (i) represents a two-terminal series-parallel contraflow network and (ii) represents an auxiliary network of (i) with ODTT.

In series-parallel graph, every cycle in the residual network has a non-negative cycle length, which is a main advantage of this graph structure. Because of this, the minimum cost circulation flow problem introduced by Ford and Fulkerson [10] for the maximum dynamic flow problem can be solved in the auxiliary network $\overline{\mathcal{N}}$. The temporally repeated $s$ - $t$ flow, thus obtained is an optimal solution to the EACF problem on a two-terminal series-parallel graph in polynomial time. So, the polynomial solution of EACF problem with ODTT by allowing the intermediate storage is possible by using Pyakurel and Dempe [24] and applying the ODTT on $\overline{\mathcal{N}}$. Moreover, for general networks, pseudo-polynomial time solution strategy exists to solve EACF problem by using time expanded network, 6, 25, 29] without permitting the intermediate storage. The EACF problem with ODTT by allowing the storage of flow at intermediate nodes in general network can be obtained by using algorithm of Hoppe and Tardos [15] in modified $s-D$ network. 


\section{Conclusion}

At the time of evacuation, improvement of flow values and reduction of the time horizon by the reversal of arcs is one of the best approaches. At the meantime, settlement of flow at intermediate nodes can be a milestone to shift the maximum evacuees from the danger zone (source). In literature, the maximum static and maximum dynamic contraflow problems with and without intermediate storage of flow have been studied in two terminal general network. Similarly, earliest arrival contraflow problem with intermediate storage has been studied in two-terminal series-parallel graph. All of these contraflow problems have been acquired symmetric transit times on the anti-parallel arcs.

In this paper, we present the flow models with asymmetric transit times on anti-parallel arcs by allowing the storage of flow at intermediate nodes. By adopting the orientation dependent transit times on the antiparallel arcs, we introduce the maximum dynamic contraflow and earliest arrival contraflow problems. We also present the polynomial time algorithms to solve the problems. Though the priority order of the nodes in our solution strategy is on the basis of distance before the contraflow configuration, our algorithms can be used for any other priority order.

Acknowledgments The first author (Durga Prasad Khanal) thanks to the German Academic Exchange Service - DAAD for Research Grants - Bi-nationally Supervised Doctoral Degrees/Cotutelle, 2021/22 and the second author (Urmila Pyakurel) thanks to the Alexander von Humboldt Foundation for Digital Cooperation Fellowship (August 1, 2021 - January 31, 2022).

\section{REFERENCES}

[1] R.K. Ahuja, T.L. Magnanti and J.B. Orlin, Network Flows: Theory, Algorithm and Applications, Prentice Hall, Englewood Cliffs, 1993.

[2] J.E. Aronson, A survey of dynamic network flows, Annals of Operations Research, vol. 20, pp. 1-66, 1998.

[3] A. Arulselvan, Network Model for Disaster Management, PhD thesis, University of Florida USA, 2009.

[4] N. Baumann, E.M. Köhler, Approximating earliest arrival flows with flow-dependent transit times, Discrete Applied Math, vol. 155, pp. 161-171, 2007.

[5] T.N. Dhamala, S.P. Gupta, D.P. Khanal, U. Pyakurel, Quickest multi-commodity flow over time with partial lane reversals, Journal of Mathematics and statistics, vol. 16, pp. 198-211, 2020.

[6] T.N. Dhamala, U. Pyakurel, Earliest arrival contraflow problem on series-parallel graphs, International Journal of Operations Research, vol. 10, pp. 1-13, 2013.

[7] T.N. Dhamala, U. Pyakurel, S. Dempe, A critical survey on the network optimization algorithms for evacuation planning problems, International Journal of Operations Research, vol. 15 no.3, pp. 101-133, 2018.

[8] E.W. Dijkstra, A note on two problems in connection with graph, Numer. Math. vol. 1, no. 1, pp. 269-271, 1959.

[9] L.R. Ford, D.R. Fulkerson, Maximal flow through a network, Canadian Journal of Mathematics, vol. 8, pp. 399-404, 1956.

[10] L.R. Ford, D.R. Fulkerson, Flows in Networks. Princeton University Press, Princeton, New jersey, 1962.

[11] D. Gale, Transient flows in networks, Michigan Mathematical Journal, vol. 6, pp. 59-63, 1959.

[12] S.P. Gupta, U. Pyakurel, T.N. Dhamala, Generalized dynamic contraflow with non-symmetric transit times, American Journal of Computational and Applied Mathematics, vol. 11, no. 1, pp. 12-17, 2021.

[13] S.P. Gupta, U. Pyakurel, T.N. Dhamala, Network flows with arc reversals and non-Symmetric transit times, American Journal of Mathematics and Statistics, vol. 11, no. 2, pp. 27-33, 2021.

[14] H.W. Hamacher, S.A. Tjandra, Mathematical modeling of evacuation problems: a state of the art, Pedestrian and Evacuation Dynamics, pp. 227-266, 2002. 
[15] B. Hoppe, E. Tardos, The quickest transshipment problem, Mathematics of Operations Research, vol. 25, pp. 36-62, 2000.

[16] D.P. Khanal, U. Pyakurel, T.N. Dhamala, Maximum Multicommodity Flow with Intermediate Storage, Mathematical Problems in Engineering, Hindawi, 2021, DOI: https://doi.org/10.1155/2021/5063207

[17] S. Kim, S. Shekhar, Contraflow network reconfiguration for evacuation planning: a summary of results, In: Proceedings of 13th ACM Symposium on Advances in Geographic Information Systems GIS, vol. 05, pp. 250-259, 2005.

[18] S. Kim, S. Shekhar, M. Min, Contraflow transportation network reconfiguration for evacuation route planning, IEEE Transactions on Knowledge and Data Engineering, vol. 20, no. 8, pp. 1115-1129, 2008.

[19] B. Kotnyek, An annotated overview of dynamic network flows, Rapport de recherche 4936, INRIA Sophia Antipolis, 2003.

[20] I.S. Kotsireas , A. Nagurney, P.M. Pardalos, Dynamics of disasters-algorithmic approaches and applications, Springer Optimization and Its Applications, 2018.

[21] E. Minieka, Maximal Lexicographic and Dynamic Network flows, Operations Research, vol. 21, pp. 517-527, 1973.

[22] H.N. Nath, U. Pyakurel, T.N. Dhamala, Network reconfiguration with orientation dependent transit times, International Journal of Mathematics and Mathematical Sciences, Hindawi, Vol. 2021, Article ID 6613622, 2021, DOI: https://doi.org/10.1155/2021/6613622.

[23] U. Pyakurel, S. Dempe, Network Flow with Intermediate Storage: Models and Algorithms, SN Operations Research Forum, 2020, DOI:10.1007/s43069-020-00033-0.

[24] U. Pyakurel, S. Dempe, Universal Maximum Flow with Intermediate Storage for Evacuation Planning, In: Kotsireas I.S., Nagurney A., Pardalos P.M., Tsokas A. (eds) Dynamics of Disasters. Springer Optimization and Its Applications, vol. 169, Springer, Cham. 2021, https : //doi.org/10.1007/978-3030 - 64973 - 9_14.

[25] U. Pyakurel, T.N Dhamala, Models and algorithms on contraflow evacuation planning network problems, International Journal of Operations Research, vol. 12, pp. 36-46, 2015.

[26] U. Pyakurel, S.P. Gupta, D.P. Khanal, T.N. Dhamala, Efficient algorithms on multi-commodity flow over time problems with partial lane reversals, International Journal of Mathematics and Mathematical Sciences, Hindawi, 2020, DOI: https://doi.org/ 10.1155/2020/2676378.

[27] S. Rebennack, A. Arulselvan, L. Elefteriadou, P.M. Pardalos, Complexity analysis for maximum flow problems with arc reversals, Journal of Combinatorial Optimization, vol. 19, pp. 200-216, 2010.

[28] S. Ruzika, H. Sperber, M. Steiner, Earliest arrival flows on series- parallel graphs Networks, vol. 10, pp. 169-173, 2011.

[29] W.L. Wilkinson, An algorithm for universal maximal dynamic flows in a network, Operations Research, vol. 19, pp. 1602-1612, 1971. 